\title{
Cut-Off Point of Waist Circumference Used for the Diagnosis of Metabolic Syndrome Among Adult Population in Ahvaz, Southwestern Iran
}

\author{
Hajieh Shahbazian ${ }^{1}$; Seyed Mahmoud Latifi ${ }^{1,}$; Sedigheh Nouhjah ${ }^{1}$ \\ ${ }^{1}$ Health Research Institute, Diabetes Research Center, Ahvaz Jundishapur University of Medical Sciences, Ahvaz, IR Iran \\ ${ }^{*}$ Corresponding author: Seyed Mahmoud Latifi, Health Research Institute, Diabetes Research Center, Ahvaz Jundishapur University of Medical Sciences, Ahvaz, IR Iran. Tel: +98-6113369539, \\ Fax: +98-6113a369539, E-mail: sml1381@yahoo.com \\ Received: 27 November 2014; Revised: 26 January 2015; Accepted: 27 February, 2015
}

\begin{abstract}
Background: Metabolic syndrome (MetS) is a well-known risk factor for type 2 diabetes and cardiovascular disease. Waist circumference (WC) is an essential component for the diagnosis of the MetS.

Objectives: The aim of this study was to compare the cut-off points of WC proposed by the international diabetes federation (IDF), adult treatment panel (ATP III), and iranian national committee of obesity (INCO) with that of the present study for predicting the MetS.

Patients and Methods: This cross-sectional study was performed on 930 individuals (439 males and 491 females) aged more than 20 years living in Ahvaz City, capital of Khuzestan Province in southwestern Iran during 2009 - 2010. Waist circumference cut-off values were obtained using a receiver operator characteristic curve. Optimal cut-off values were calculated by plotting the true-positive rate against the false-positive rate. Also, sensitivity, specificity, and Youden's Index were calculated.

Results: The cut-off points of WC, according to a maximum sum of sensitivity plus specificity for detecting at least two components of MetS were found to be $91.5 \mathrm{~cm}$ and $85.5 \mathrm{~cm}$ in men and women, respectively. Using these values, sensitivity and specificity were $61 \%$ in men and $65 \%$ in women. The sensitivity of the male population and the specificity of the female population were found to be higher than the values proposed by IDF and ATP III.

Conclusions: Optimal WC values for defining MetS were $91.5 \mathrm{~cm}$ and $85.5 \mathrm{~cm}$ in men and women, respectively. The cut-off values recommended by world organizations are not appropriate for an adult population living in Ahvaz City.
\end{abstract}

Keywords: Waist Circumference; Metabolic Syndrome X; Receiver Operator Characteristic Curve

\section{Background}

Metabolic syndrome (MetS) as a global public health problem is a complex constellation of metabolic abnormalities including hypertension, elevated triglycerides, decreased high-density lipoprotein (HDL), hyperglycemia and central obesity (1). Metabolic syndrome, also known as syndrome X (2), is associated with 3 fold and 2 fold increases in type 2 diabetes mellitus and cardiovascular disease, respectively (3). Cardiovascular diseases are among the main causes of mortality in Iran (4). Several definitions, using different criteria for MetS have been proposed since 1998 (5). The first definition was released by the world health organization (WHO) based on laboratory measurement of insulin resistance and the existence of two components of the following risk factors: lowdensity lipoprotein (LDL) cholesterol, obesity, high blood pressure and elevated triglycerides. The third adult treatment panel (ATP III) defined the syndrome based on the presence of 3 of 5 criteria including LDL and HDL levels, fasting hyperglycemia, hypertension, hypertriglyceridemia and central obesity with an emphasis on cardiovascular hazards. Central obesity was defined as a WC of more than 102 and 88 for men and women, respectively. International diabetes federation (IDF) generated new definition and decreased cut-off values for WC to 94 and 80 for men and women, respectively $(6,7)$.

The Iranian National Committee of Obesity (INCO) survey found that a WC cut-off point of $90 \mathrm{~cm}$ is a predictor of MetS in both genders (8). Central obesity, as assessed by WC, based on IDF and national cholesterol education program's adult treatment (NCEP III) definitions, is an important component of MetS $(6,7)$. Determination of cut-off points for WC is accepted as the best anthropometric indicator of abdominal adiposity, which reflects increased abdominal fat or visceral fat, which has an essential role in pathogenesis of MetS (9-14). Waist circumference has been shown to be more correlated with MetS components better than fat percentage (15).

So much evidence shows a link between WC and other criteria of MetS and cardiovascular disease (16). Waist circumference is a simple valid, cost effective clinical anthropometric measure predictor for detection of MetS (918). The use of WC is preferred to BMI for evaluation of abdominal adiposity (19), but the cut-off values of WC vary according to ethnicity, gender and lifestyle; therefore, population-specific cutoff points were incorporated into the definition $(6,20)$.

Copyright (C) 2015, Ahvaz Jundishapur University of Medical Sciences. This is an open-access article distributed under the terms of the Creative Commons Attribution-NonCommercial 4.0 International License (http://creativecommons.org/licenses/by-nc/4.0/) which permits copy and redistribute the material just in noncommercial usages, provided the original work is properly cited. 


\section{Objectives}

Therefore, obtaining appropriate uniform WC cut-off value of the adult population has an essential role in diagnosis of MetS. The aim of the present study was to determine the optimal WC cut-off values in adult population living in Ahvaz city and compare the results with those of the IDF, ATP III and INCO as a predictor of MetS.

\section{Patients and Methods}

This cross-sectional study was conducted by the diabetes research center of Ahvaz Junsdishapur University of Medical Sciences on 930 adults (age range, more than 20 years) referred to 6 urban health centers in Ahvaz city, capital of Khuzestan Province in southwestern Iran during 2009 - 2010. The cluster random sampling technique was used to select the samples. In the selected health center, 75 households were selected randomly.

Blood pressure of participants was measured in a sitting position after 15 minutes rest by a standard sphygmomanometer. The cut-off was located on the right arm at the level of the heart of the participant. Blood pressure was measured two times with at least a 30-minute interval. Waist circumference was measured at the midpoint between the lowest rib and the upper lateral border of the right iliac crest and hip circumference at the point of maximum hip diameter.

Details of other anthropometric indices measured by biochemical and clinical tests were reported elsewhere (21). A structured questionnaire was used for the collection of demographic data, anthropometric measures and risk factors of cardiovascular disease. The prevalence of MetS among participants was determined based on IDF, ATP III and INCO criteria. The optimal cut-off values of WC for detecting at least 2 components were calculated using a receiver operating characteristic (ROC) curve.

ROC curves were calculated by plotting sensitivity (the true positive rate) against 1-specificity (the false-positive rate), which maximal accuracy was gained. Also, sensitivity (ability to identify true cases correctly), specificity (ability to identify true no-cases correctly) and Youden's Index (sensitivity + specificity-1) were calculated. The Youden's Index is the maximum difference between the ROC curve and the diagonal line. Values close to 1 indicate that the biomarker's ability is high (22). Data were analyzed using chi-square test and Independent t-test with SPSS Inc, Chicago, IL, USA. A P $<0.05$ was considered as statistically significant.

\section{Results}

A total of 930 adults (439 males and 491 females) aged over 20 years were studied. The mean age of the participant was $42.27 \pm 14$ years ( $44.2 \pm 14.2$ years for men and $40.5 \pm 13.5$ years for women).

The prevalence rates of diabetes mellitus were $14.5 \%$ in men and $11.7 \%$ in women. The prevalence rates of hypertension, high triglyceride and low HDL cholesterol were $8.7 \%, 47.1 \%$, and $28.3 \%$ in men while they were $2.9 \%, 34.1 \%$, and $50.2 \%$ in women, respectively.

The prevalence rates of MetS were $21.8 \%$ and $27.4 \%$ based on ATP III and IDF criteria, respectively. The prevalence rates of MetS were estimated to be $28.9 \%$ and $31.7 \%$ in women, but $14 \%$ and $22.6 \%$ in men based on ATP III and IDF criteria, respectively).

The optimal cut-off point of WC for predicting MetS with at least two other components (except WC) was $91.5 \mathrm{~cm}$ for men and $85.5 \mathrm{~cm}$ for women. Table 2 shows the Area under the Curve (AUC) for WC, sensitivity, and specificity according to two international diabetes organizations, INCO and the the current study based on gender of the participants. Cut-off point of WC, sensitivity, specificity, positive likelihood ratio and prevalence of MetS, according to two international diabetes organizations, INCO and the Ahvaz study are presented in Table 3. For the male and female participants with two or more MetS components, the areas under the ROC curve were 0.72 (95\% CI, $0.67-0.76)$ and 0.646 (0.59-0.69), respectively.

The ROC curves for WC to detect the presence of at least two risk factors of the MetS in subjects of both genders are presented in Figure 1 and 2.

\begin{tabular}{lccc}
\hline \multicolumn{1}{l}{ Table 1. Characteristic of the Population Studied According to Metabolic Syndrome (Component by Sex) a,b,c } \\
\hline Variable & Women $(\mathbf{n}=\mathbf{4 9 1})$ & Men $(\mathbf{n}=\mathbf{4 3 9})$ & P Value \\
\hline Age, $\mathbf{y}$ & $40.55 \pm 13.5$ & $44.2 \pm 14.2$ & 0.0001 \\
WC, $\mathbf{~ m ~}$ & $85 \pm 12.3$ & $90.8 \pm 10.7$ & 0.0001 \\
FBG, $\mathbf{~ m m o l} / \mathbf{L}$ & $5.74 \pm 2.22$ & $6.06 \pm 2.58$ & 0.047 \\
TG, $\mathbf{m m o l} / \mathbf{L}$ & $1.68 \pm 1.21$ & $2.02 \pm 1.27$ & 0.0001 \\
HDL, $\mathbf{m m o l} / \mathbf{L}$ & $1.58 \pm 0.32$ & $1.37 \pm 0.9$ & 0.0001 \\
\hline Systolic b.p., $\mathbf{~ m m ~ H g}$ & $114 \pm 21.4$ & $116.2 \pm 20.6$ & 0.11 \\
\hline Diastolic b.p., $\mathbf{~ m m ~ H g}$ & $70.1 \pm 15.3$ & $72.4 \pm 15.2$ & 0.02 \\
\hline
\end{tabular}

\footnotetext{
a Abbreviations: FBG, fasting plasma glucose; HDL, high-density lipoprotein; TG, triglyceride;.WC, waist circumference

$\mathrm{b}$ Data is presented as mean \pm SD.

c P values were determined using an Independent t-test.
} 


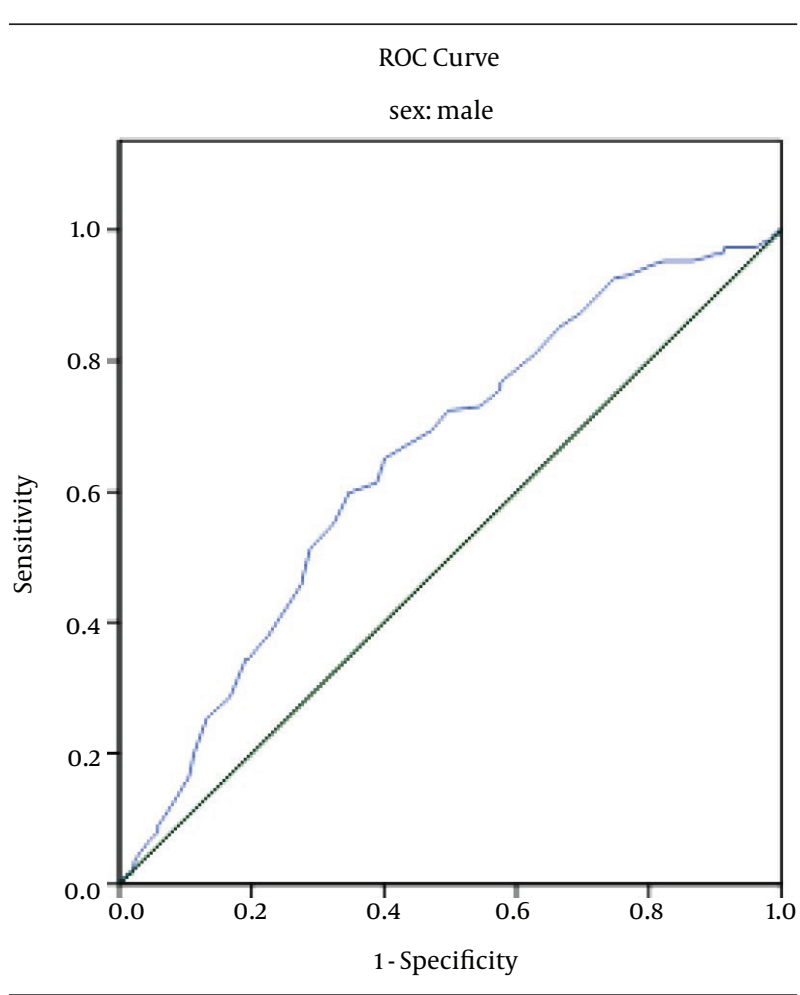

Figure 1. The ROC Curves for WC to Detect the Presence of at Least Two Risk Factors of the Metabolic Syndrome (MetS) in Male Subjects

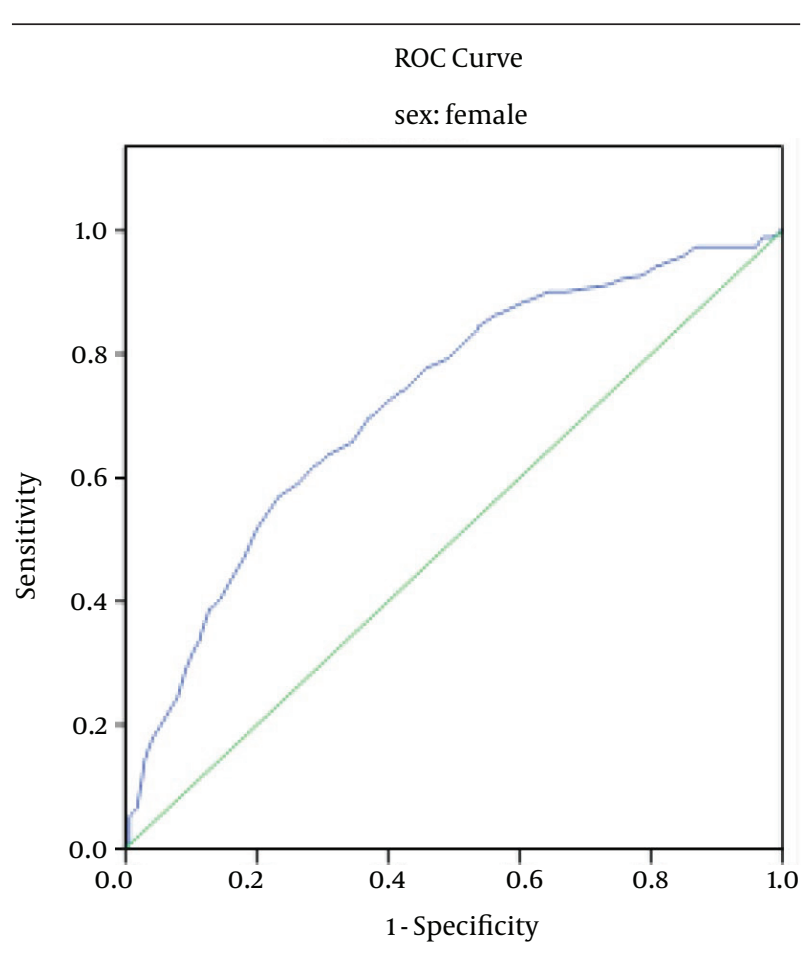

Figure 2. The ROC Curves for WC to Detect the Presence of at Least Two Risk Factors of the (MetS) Metabolic Syndrome in Female Subjects

Table 2. The Area Under the Curve for Waist Circumference, Sensitivity, and Specificity According to Two International Diabetes Organizations, National Iranian Committee of Obesity and the Ahvaz Study Based on Gender ${ }^{\text {a }}$

\begin{tabular}{lccccc}
\hline Variables & Our Cut-Off(AUC) & Our Cut- Off & IDF & ATP III & INCO \\
\hline Men & & & & & \\
\hline High blood pressure & $93.5(0.665)$ & $0.665,0.62$ & $0.61,0.66$ & $0.23,0.87$ & $0.73,0.47$ \\
\hline Hyperglycemia & $91.5(0.595)$ & $0.55,0.57$ & $0.50,0.64$ & $019,0.88$ & $0.63,0.49$ \\
\hline HDL $<40$ & $91.5(0548)$ & $0.55,0.55$ & $0.49,0.61$ & $0.19,0.87$ & $0.61,0.46$ \\
\hline Triglycerides $>150$ & $90.5(0.674)$ & $0.65,0.64$ & $0.53,0.71$ & $0.18,0.89$ & $0.70,0.59$ \\
\hline$\geq 2$ of the Above & $91.5(.646)$ & $0.61,0.61$ & $0.55,0.68$ & $0.20,0.89$ & $0.69,0.53$ \\
\hline Women & & & & $0.67,0.64$ & $0.61,0.69$ \\
\hline High blood pressure & $87.75(0.68)$ & $0.68,0.66$ & $0.86,0.39$ & $0.59,0.68$ & $0.53,0.73$ \\
\hline Hyperglycemia & $85.5(0.664)$ & $0.62,0.62$ & $079,0.43$ & $0.44,0.61$ & $0.38,0.66$ \\
\hline HDL $<40$ & $84.25(0.547)$ & $0.54,0.53$ & $0.70,0.40$ & $0.61,0.68$ & $0.56,0.73$ \\
\hline Triglycerides $>150$ & $85.5(0.73)$ & $0.67,0.63$ & $0.86,0.45$ & $0.62,0.72$ & $0.57,0.77$ \\
\hline$\geq 2$ of the Above & $85.5(0.721)$ & $0.65,0.65$ & $0.83,0.46$ & \\
\hline
\end{tabular}

a Abbreviations: ATP III, adult treatment panel, AUC, under the curve; HDL, high-density lipoproteins; IDF, international diabetes federation; INCO, Iranian national committee of obesity criteria

\section{Discussion}

The IDF proposed that central obesity, as assessed by WC cut-off values specific to the ethnicity and gender, is a prominent component of MetS (6).

In the present study, the optimal cut-off values of WC for diagnosis of MetS were calculated to be $91.5 \mathrm{~cm}$ and 85.5 $\mathrm{cm}$ for the male and female adult population of Ahvaz city. These cut-off values are the same as those proposed by Esteghamati et al. in Tehran, capital of Iran, with higher sensitivity (77\% in men and $88 \%$ in women) and lower specificity (58\% in men and 50\% in women) in comparison to our study (22). Kaykhaei et al. (2012) proposed cutoff point of WC to be $93.5 \mathrm{~cm}$ for men (sensitivity $=59.27 \%$, 
Table 3. Cut-Off Point of Waist Circumference, Sensitivity, Specificity and Prevalence of Metabolic Syndrome According to Two International Diabetes Organizations, Iranian National Committee of Obesity criteria (INCO) and the current study ${ }^{\text {a }}$

\begin{tabular}{|c|c|c|c|c|c|}
\hline Index & WC & Sensitivity & Specificity & Youden's Index & Prevalence of MetS \\
\hline \multicolumn{6}{|l|}{ IDF } \\
\hline Men & 94 & 0.55 & 0.68 & 0.23 & 22.6 \\
\hline Women & 80 & 0.83 & 0.46 & 0.14 & 31.7 \\
\hline \multicolumn{6}{|l|}{ ATP III } \\
\hline Men & 102 & 0.2 & 0.89 & 0.09 & 0.14 \\
\hline Women & 88 & 0.62 & 0.72 & 0.34 & 28.9 \\
\hline \multicolumn{6}{|l|}{ Ahvaz } \\
\hline Men & 91.5 & 0.6 & 0.61 & 0.22 & 22.6 \\
\hline Women & 85.5 & 1.65 & 0.65 & 0.3 & 28.9 \\
\hline \multicolumn{6}{|l|}{ INCO } \\
\hline Men & 90 & 0.69 & 0.53 & 0.22 & 30.7 \\
\hline Women & 90 & 0.57 & 0.77 & 0.34 & 26.7 \\
\hline
\end{tabular}

a Abbreviations: IDF, international diabetes federation; ATP III, adult treatment panel; INCO, Iranian national committee Of obesity criteria

specificity $=78.85 \%$ ) and $85.5 \mathrm{~cm}$ for women (sensitivity = $75.79 \%$, specificity $=62.78 \%$ ) in Zahedan, southeast of Iran (23). Sharifi et al. (2008) calculated optimal cut-off point of WC, $87 \mathrm{~cm}$ for males (sensitivity $=59.9 \%$, specificity = $69.5 \%$ ) and $82 \mathrm{~cm}$ for females (sensitivity $=68.7 \%$, specificity $=68 \%$ ) according to the Zanjan study (24).

One study yielding results similar to the present study is, Lee et al. (2007) which proposed cut-off points of WC for predicting two components of MetS to be 90 $\mathrm{cm}$ for males and $85 \mathrm{~cm}$ for females suggesting them as appropriate WC cut-off points for central obesity for Korean adults (25).

Moreover, WHO has defined normal WC to be $102 \mathrm{~cm}$ for men and $88 \mathrm{~cm}$ for women (26). In Iraq, the cut-off point based on the maximum sensitivity and specificity for predicting the MetS was calculated to be $97 \mathrm{~cm}$ for men and 99 $\mathrm{cm}$ for women. The sensitivity and specificity of these values were $70 \%$ and $50 \%$ in men, and $70 \%$ and $45 \%$ in women, respectively (5). Waist circumference cut-off point's variation proposed by different studies may suggest the importance of lifestyle factors plus racial differences (19).

In present study, cut-off value of WC was found to be higher in men. Similar to our findings, IDF (6), ATP III (7) and WHO (26) proposed higher WC for men. Moreover our results are in line with the Tehran study (21). Contrary to our findings, Japan (27) Oman (28) and Iraq (5) studies reported higher cut-off values for detecting MetS in females. Also, Abbaszadeh-Ahranjani et al. in a national report proposed higher cut-off points for women compared to men (29).

In comparison with IDF, which proposed a WC of 94 $\mathrm{cm}$ for men and $80 \mathrm{~cm}$ for women as normal values, sensitivity was higher in men (61\% versus $55 \%$ ) and lower in women (65\% versus $94 \%$ ). Higher sensitivity in men and higher specificity in women were observed compared to ATP III definition.
As can be seen in Table 3 the Youden's index WC cut-off point value proposed by IDF for women (0.14) is very poor as is the case with ATP III's proposed index for men (0.09). Therefore, these indices cannot be considered useful for evaluations conducted in Iran. The Youden's indices obtained in INCO and Ahvaz study are very close ( 0.22 for Men, 0.34 and 0.3 for women, respectively) but the difference is that the Youden's index in the Ahvaz study has the optimum values for both sensitivity and specificity while those of INCO study are unbalanced.

Optimal WC values for detecting MetS were found to be $91.5 \mathrm{~cm}$ and $85.5 \mathrm{~cm}$ for males and females, respectively. The cut-off values recommended by world organizations are not appropriate for an adult population living in Ahvaz City. Due to the high prevalence of MetS, achieving an agreed upon definition for WC must be considered a priority.

\section{Acknowledgements}

This paper is issued from the research project (D-8701). The authors would like to thanks all staff of diabetes research center of Ahvaz Jundishapur University of Medical Sciences, especially Miss Reshadatian and Dehghan, for their help in this study.

\section{Authors' Contributions}

All of authors contributed equally to this work.

\section{Funding/Support}

Financial support was provided by diabetes research center, Ahvaz Jundishapur University of Medical Sciences.

\section{References}

1. Magliano DJ, Shaw JE, Zimmet PZ. How to best define the metabolic syndrome. Ann Med. 2006;38(1):34-41. 
2. Reaven GM. Banting Lecture 1988. Role of insulin resistance in human disease. 1988. Nutrition. 1997;13(1):65.

3. Zimmet P, Magliano D, Matsuzawa Y, Alberti G, Shaw J. The metabolic syndrome: a global public health problem and a new definition. JAtheroscler Thromb. 2005;12(6):295-300.

4. Sarraf-Zadegan N, Boshtam M, Malekafzali H, Bashardoost N, Sayed-Tabatabaei FA, Rafiei M, et al. Secular trends in cardiovascular mortality in Iran, with special reference to Isfahan. Acta Cardiol. 1999;54(6):327-33.

5. Mansour AA, Al-Hassan AA, Al-Jazairi MI. Cut-off values for waist circumference in rural Iraqi adults for the diagnosis of metabolic syndrome. Rural Remote Health. 2007;7(4):765.

6. Alberti KG, Zimmet P, Shaw J. Metabolic syndrome--a new worldwide definition. A Consensus Statement from the International Diabetes Federation. Diabet Med. 2006;23(5):469-80.

7. National Cholesterol Education Program Expert Panel on Detection E, Treatment of High Blood Cholesterol in A. Third Report of the National Cholesterol Education Program (NCEP) Expert Panel on Detection, Evaluation, and Treatment of High Blood Cholesterol in Adults (Adult Treatment Panel III) final report. Circulation. 2002;106(25):3143-421.

8. Azizi F, Hadaegh F, Khalili D, Esteghamati A, Hosseinpanah F, Delavari A, et al. Appropriate definition of metabolic syndrome among Iranian adults: report of the Iranian National Committee of Obesity. Arch Iran Med. 2010;13(5):426-8.

9. World Health Organization. Physical status: the use and interpretation of anthropometry. WHO technical report series no 854. Geneva : S1995. Available from: http://www.who.int/childgrowth/publications/physical_status/en/.

10. Liu KH, Chan YL, Chan WB, Kong WL, Kong MO, Chan JC. Sonographic measurement of mesenteric fat thickness is a good correlate with cardiovascular risk factors: comparison with subcutaneous and preperitoneal fat thickness, magnetic resonance imaging and anthropometric indexes. Int J Obes Relat Metab Disord. 2003;27(10):1267-73.

11. Wannamethee SG, Shaper AG, Morris RW, Whincup PH. Measures of adiposity in the identification of metabolic abnormalities in elderly men. Am J Clin Nutr. 2005;81(6):1313-21.

12. Lean ME, Han TS, Morrison CE. Waist circumference as a measure for indicating need for weight management. BMJ. 1995;311(6998):158-61.

13. Han TS, McNeill G, Seidell JC, Lean ME. Predicting intra-abdominal fatness from anthropometric measures: the influence of stature. Int J Obes Relat Metab Disord. 1997;21(7):587-93.

14. Despres JP, Prud'homme D, Pouliot MC, Tremblay A, Bouchard C. Estimation of deep abdominal adipose-tissue accumulation from simple anthropometric measurements in men. Am J Clin Nutr. 1991;54(3):471-7.

15. Shen W, Punyanitya M, Chen J, Gallagher D, Albu J, Pi-Sunyer X, et al. Waist circumference correlates with metabolic syndrome indicators better than percentage fat. Obesity (Silver Spring). 2006;14(4):727-36.

16. Pouliot MC, Despres JP, Lemieux S, Moorjani S, Bouchard C, Tremblay A, et al. Waist circumference and abdominal sagittal diam- eter: best simple anthropometric indexes of abdominal visceral adipose tissue accumulation and related cardiovascular risk in men and women. Am J Cardiol. 1994;73(7):460-8.

17. Seidell JC, Kahn HS, Williamson DF, Lissner L, Valdez R. Report from a Centers for Disease Control and Prevention Workshop on use of adult anthropometry for public health and primary health care. Am JClin Nutr. 2001;73(1):123-6.

18. Zhu S, Wang Z, Heshka S, Heo M, Faith MS, Heymsfield SB Waist circumference and obesity-associated risk factors among whites in the third National Health and Nutrition Examination Survey: clinical action thresholds. Am J Clin Nutr. 2002;76(4):743-9.

19. Dalton M, Cameron AJ, Zimmet PZ, Shaw JE, Jolley D, Dunstan DW et al. Waist circumference, waist-hip ratio and body mass index and their correlation with cardiovascular disease risk factors in Australian adults. JIntern Med. 2003;254(6):555-63.

20. Blumel JE, Legorreta D, Chedraui P, Ayala F, Bencosme A, Danckers L, et al. Optimal waist circumference cutoff value for defining the metabolic syndrome in postmenopausal Latin American women. Menopause. 2012;19(4):433-7.

21. Shahbazian H, Latifi SM, Jalali MT, Shahbazian H, Amani R, Nikhoo A, et al. Metabolic syndrome and its correlated factors in an urban population in South West of Iran. J Diabetes Metab Disord. 2013;12(1):11.

22. Esteghamati A, Ashraf H, Rashidi A, Meysamie A. Waist circumference cut-off points for the diagnosis of metabolic syndrome in Iranian adults. Diabetes Res Clin Pract. 2008;82(1):104-7.

23. Kaykhaei M, Hashemi M, Narouie B, Shikhzadeh A, Jahantigh M, Shirzaei E, et al. Prevalence of metabolic syndrome in adult population from zahedan, southeast iran. Iran J Public Health. 2012;41(2):70-6.

24. Sharifi F, Mousavinasab N, Mazloomzadeh S, Jaberi Y, Saeini M, Dinmohammadi M, et al. Cutoff point of waist circumference for the diagnosis of metabolic syndrome in an Iranian population. Obesity Res Clin Prac. 2008;2(3):171-8.

25. Lee SY, Park HS, Kim DJ, Han JH, Kim SM, Cho GJ, et al. Appropriate waist circumference cutoff points for central obesity in Korean adults. Diabetes Res Clin Pract. 2007;75(1):72-80.

26. WHO . Diet, nutrition and the prevention of chronic diseases. Geneva:s 2003. Available from: http://www.fao.org/DOCREP/005/ AC911E/AC911E00.HTM.

27. Oka R, Kobayashi J, Yagi K, Tanii H, Miyamoto S, Asano A, et al. Reassessment of the cutoff values of waist circumference and visceral fat area for identifying Japanese subjects at risk for the metabolic syndrome. Diabetes Res Clin Pract. 2008;79(3):474-81.

28. Al-Lawati JA, Jousilahti P. Body mass index, waist circumference and waist-to-hip ratio cut-off points for categorisation of obesity among Omani Arabs. Public Health Nutr. 2008;11(1):102-8.

29. Abbaszadeh-Ahranjani S, Kashani H, Mohajeri-Tehrani MR, Larijani B, Forouzanfar MH, Afshani $\mathrm{H}$. Clinical research Cut-oft points of waist circumference and body mass index for detecting diabetes, hypercholesterolemia and hypertension according to National Non-Communicable Disease Risk Factors Surveillance in Ira. Arch Med Sci. 2012;8(4):614-21. 